\title{
Molecular detection of Leishmania parasites from whole bodies of sandflies collected in Nepal
}

\author{
Kishor Pandey ${ }^{1 *}$, Shisir Pant ${ }^{2}$, Hiroji Kanbara ${ }^{1}$, Mohammed Nasir Shuaibu ${ }^{3}$, Arun Kumar Mallik ${ }^{4}$, \\ Basu Dev Pandey ${ }^{5}$, Osamu Kaneko ${ }^{1}$, Tetsuo Yanagi ${ }^{6}$
}

${ }^{1}$ Department of Protozoology, Institute of Tropical Medicine, Nagasaki University, 1-12-4 Sakamoto, Nagasaki 852-8523, Japan Tel: (+81)-95-819-7838, Fax: (+81)-95-819-7805, email: pandey_kishor@hotmail.com

${ }^{2}$ Vector Borne Disease Research and Training Center, Hetauda, Nepal

${ }^{3}$ Department of Immunogenetics, Institute of Tropical Medicine, Nagasaki University, 1-12-4 Sakamoto, Nagasaki 852-8523, Japan

${ }^{4}$ Janakpur Zonal Hospital, Janakpur, Nepal

${ }^{5}$ Sukraraj Tropical and Infectious Diseases Hospital, Kathmandu, Nepal

${ }^{6}$ Animal Research Center for Tropical Infections, Institute of Tropical Medicine, Nagasaki University, 1-12-4 Sakamoto, Nagasaki 852-8523, Japan

\begin{abstract}
Visceral leishmaniasis is endemic in the southern part of the Terai region of Nepal. Natural infections of Phlebotomus species with Leishmania parasites in these endemic areas were analyzed by a polymerase chain reaction (PCR) amplification based assay. A total of 401 Phlebotomus argentipes and $202 \quad$ P. papatasi female sandflies were captured in the Dhanusa district from 2004 to 2006, and analyzed. It was found that $6.7 \%$ of P. argentipes, but no P. papatasi were positive for Leishmania parasites, suggesting that $P$. argentipes is a major vector in these areas. The infectivity of $P$. argentipes with Leishmania was consistent with infection rates reported from other areas of the world. This is the first report of naturally infected Leishmania parasites in sandflies collected from Nepal.
\end{abstract}

Keywords: Phlebotomus, infectivity, visceral leishmaniasis, Nepal

\section{Introduction}

Leishmaniasis is caused by protozoan parasites belonging to the genus Leishmania. The parasites are distributed worldwide, especially in tropical and subtropical areas, and affect at least 12 million people. More than 20 species of Leishmania have been described as causative agents of human leishmaniases. (Desjeux 1996, Choi and Lerner 2001). Leishmania parasites are transmitted by female sandflies of the genus Phlebotomus in the Old World and Lutzomyia in the New World. The spread of leishmaniases depends on the distribution of vectors and reservoir animals. There are more than 500 sandfly species, but only 20 are of medical importance. In addition, it has been suggested that only restricted sandfly species can support the development of specific Leishmania parasites (Killick-Kendrick 1999). Thus, the detection and identification of Leishmania species within naturally infected sandflies is important for the prediction of the risk and expansion of the disease in endemic areas. In Nepal, visceral leishmaniasis (VL) is confined mainly to the southern parts of the Terai region, which borders the VL endemic districts of Bihar state in India. Around 5.5 million people live in the affected areas, and those people are classified as "at risk". A total of 25,704 cases with 530 deaths were reported between 1980 and 2004, and the fatality rate in reported cases varied between 0.23 and 13.6\% (Ministry of Health, Nepal 2005/2006).

Phlebotomus argentipes are much more abundant than $P$. papatasi and Sergentomyia spp. in India (Dinesh et al. 2000, Mukherjee et al. 1997). P. argentipes is largely restricted to domestic and peri-domestic habitats and a limited number of studies have detected breeding sites in humid ground rich in organic content mainly in animal shelters (Palit et al. 2005). It is important to survey the infection rate of naturally infected sandflies in endemic areas. However, studies based on the demonstration of promastigotes in dissected sandflies by microscopy are difficult to carry out, as the infection rate in the competent vectors is generally low and its estimation requires examination of a large number of specimens. Previous microscopical surveys on different species of sandfly in different regions showed infection rates ranging from 1.7 to $10.7 \%$ (Bettini et al. 1986; Maroli et al. 1994b). Recently, polymerase chain reaction (PCR)-based techniques have been adapted to detect Leishmania spp. in sandflies. By semi-nested PCR assay, Aransay et al. (2000) found a high rate of $L$. infantum infection $(8.3 \%)$ in $P$. papatasi in Greece. Similarly in Argentina, Corbado-Launus et al. (2006) reported a 9.1\% positive rate for Leishmania in Lutzomyia neivai. In this study we employed a PCR method to directly detect Leishmania parasites from individual sandflies collected from endemic areas of Nepal.

\section{Material and methods}

Study sites

Dhanusa district is located in the southern part of the Terai region in Nepal, $500 \mathrm{~km}$ from Kathmandu, possessing many ponds, a small dam and dispersed dwellings with domestic animals such as dogs, goats, cows, water buffaloes, hogs and poultry. Sandflies were collected at 5 Village Development Committees (VDC; Yagyabhumi, Bateshwor, Umaprempur, Godar and Sinurjoda) of Dhanusa district (Fig 1). These 5 areas were chosen based on previously reported cases of leishmaniasis (Ministry of Health, Nepal 2005/6).

Sandflies were captured with mouth aspirators, test tubes and the help of battery-operated torches in the evening ( $3 \mathrm{hrs}$ ) and early in the morning ( $3 \mathrm{hrs}$ ) from two different places: inside houses, and in animal shelters, in August 2004, June 2005 and July 2006. On the day, the collected sandflies were brought to the field laboratory, anaesthetized with chloroform, and morphologically 
examined for species identification using criteria given by Lewis (Lewis 1987). The sandflies were fixed in $70 \%$ ethanol and transported to Japan for PCR analysis.

\section{Leishmania donovani}

Leishmania donovani (D10 isolate) was maintained as previously reported [Pandey et al 2007].

\section{DNA extraction}

Prior to DNA extraction, legs and wings of each sandfly were removed under a binocular microscope and individual female sandflies were macerated in a micro tube using a plastic pestle. DNA was extracted independently from each sandfly using the DNeasy ${ }^{B}$ Blood \& Tissue Kit (Qiagen, Valencia, CA). Finally DNA was resuspended in $100 \mu \mathrm{l}$ of Tris-EDTA buffer $(10 \mathrm{mM}$ Tris-Cl, $0.5 \mathrm{mM}$ EDTA, pH 9.0). In the absence of laboratory reared female sandflies, field-caught male sandflies were used as a control, as they do not serve as vectors for Leishmania parasites.

\section{PCR amplification}

Leishmania parasites were detected by PCR amplification of a variable region of the minicircle kinetoplast DNA as previously described (Aransay et al. 2000). Copy number of minicircle kinetoplast DNA is more than $10^{4}$ per parasite, maximizing the possibility of detection (Smyth et al 1992; Salotra et al 2001). PCR analysis consisted of two steps. First round PCR amplification was carried out in a total of $10 \mu \mathrm{l}$ reaction mixture containing $10 \mathrm{mM}$ Tris- $\mathrm{HCl}$ (pH8.3), $50 \quad \mathrm{mM} \mathrm{KCl}, \quad 250 \mu \mathrm{M}$ deoxynucleotide triphosphates, $1.5 \mathrm{mM} \mathrm{MgCl}_{2}$, 1 unit of TaKaRaTaq ${ }^{\mathrm{TM}}$ DNA polymerase (TaKaRa, Japan), $1 \mu \mathrm{M}$ LINR4 primer (5'-GGGGTTGGTGTAAAATAGGG-3'), $0.2 \mu \mathrm{M}$ LIN17 primer (5'-TTTGAACGGGATTTCTG-3') and $1 \mu \mathrm{l}$ of DNA solution. The mixture was incubated at $94^{\circ} \mathrm{C}$ for 5 min followed by 17 cycles, each consisting of $30 \mathrm{sec}$ at $94^{\circ} \mathrm{C}, 30 \mathrm{sec}$ at $52^{\circ} \mathrm{C}$ and $1 \mathrm{~min}$ at $72^{\circ} \mathrm{C}$. After the last cycle, the extension was continued for a further $5 \mathrm{~min}$. The second round semi-nested PCR was carried out in a $20 \mu \mathrm{l}$ reaction mixture volume as described for the first round by $\begin{array}{lllll}\text { adding } & 1 & \mu \mathrm{M} & \text { LIN19 } & \text { primer }\end{array}$ (5'-CAGAACGCCCCTACCCG-3') and $1 \mu \mathrm{l}$ of first PCR product for 33 cycles with the final extension at $72^{\circ} \mathrm{C}$ for $10 \mathrm{~min}$. Ten $\mu \mathrm{l}$ of the PCR-amplified products was subjected to a $2 \%$ agarose gel electrophoresis (LO3; TaKaRa), stained with ethidium bromide $(0.5 \mu \mathrm{g} / \mathrm{ml})$, and visualized under ultraviolet transillumination.

\section{Sequencing}

DNA fragments were recovered from agarose gels after electrophoresis and purified using QIAquick gel extraction kit (Qiagen). Purified DNA fragments were directly sequenced using an ABI Prism BigDye ${ }^{\circledR}$ Termanitor v1.1 Cycle Sequencing Kit (Applied Biosystem, USA) with the LIN 19 primer according to the manufacturer's instruction.

\section{Results}

Sensitivity of the PCR assay
To establish a reliable detection system for Leishmania DNA, we initially tested the specificity and sensitivity of the PCR method with primers specific for Leishmania minicircle kinetoplast DNA. We could detect parasite DNA from a sample prepared from a (theoretically) single L. donovani culture parasite (data not shown). We subsequently tested PCR sensitivity against samples prepared from different numbers of culture parasites mixed with a single male sandfly. We found that a single parasite could be detected when mixed with the body of male sandfly (Fig. 2).

Identification of Leishmania parasites from sandflies collected in Nepal

A total of 671 female sandfly specimens were collected between 2004 and 2006 in 5 different sites in Nepal (Table 1). Four hundred and one were P. argentipes (59.8\%), 202 were $P$. papatasi $(30.1 \%)$, and 68 were Sergentomyia spp. (10.1\%). Twenty seven out of $401 P$. argentipes showed positive bands ( 720 bp) by Leishmania spp. specific PCR assay, indicating that at least $6.7 \%$ were naturally infected with Leishmania parasites (Table 2). However, parasites were not detected from $P$. papatasi $(0 / 202)$ in this survey. Thus, $P$. argentipes appears to be the predominant vector for Leishmania parasites in these areas. All of the visualized bands showed the same size as standard $L$. donovani originating from Nepal (Fig. 3; Pandey et al, 2007). The PCR products were directly sequenced and compared with known sequences for species-identification within genus Leishmania. To this end, 15 samples were identified as $L$. donovani based on the strong homology with reported $L$. donovani kDNA sequence (GenBank accession number Y11401.1; Basselin et al. 1998). The remaining samples could be categorized only as Leishmania spp. based on the positive amplification with the Leishmania-specific primer set. However, six parasites showed sequences distinct from known Leishmania spp. and reliable sequences were not obtained from the remaining six samples.

\section{Discussion}

In this report, we used a PCR-based system for the detection of $L$. donovani parasites in sandflies without the need for dissection. In previous studies, PCR identification assays were carried out on parasites collected from sandflies after dissection (Aransay et al. 2000, Kato et al. 2005). In order to avoid this technically demanding and time-consuming dissection step we explored a novel strategy to amplify parasite DNA from the whole bodies of collected sandflies. The use of male sandfly mixtures spiked with different concentrations of $L$. donovani promastigotes permitted the sensitivity of the detection system to be evaluated. We have demonstrated that a single Leishmania spp. parasite may be detected by this technique, even in the presence of excess phlebotomine DNA.

The results presented here constitute the first natural infection analysis using a DNA-based approach to identify phlebotomines involved in the transmission of $\mathrm{VL}$ in Nepal. Leishmania DNA was detected only from $P$. argentipes, but not from $P$. papatasi, suggesting that $P$. argentipes is a major vector for $L$. donovani parasite in the survey areas in Nepal. Although we could not detect 
Leishmania DNA from $P$. papatasi, it is reported to be a vector for L. infantum (Aransay et al 2000) and L. major (Ready et al 1998) in other regions.

A prevalence of $6.7 \%$ for $P$. argentipes infection represents a relatively high index compared with other studies that use traditional dissection method (Table 3). We consider that this is because of the high sensitivity of PCR-based detection systems. The occurrence of laboratory contamination can be ruled out because of the lack of the amplification from negative control samples. However, it should be noted that Leishmania DNA-positive sandflies may include not only those carrying live promastigotes, but also those carrying DNA fragments of Leishmania resulting from non-established (abortive) infections or blood meals containing Leishmania DNA fragments (Gradoni, 2002).

More information regarding the distribution of this parasite in the phlebotomine fauna is essential in order to understand leishmaniasis epidemiology and to develop effective control measures against the disease. The PCR-based detection technique described here, which by-passes the need for sandfly dissection, provides a valuable tool for the analysis of large numbers of sandfly samples for endemic areas, and will greatly aid future epidemiological studies.

\section{Acknowledgements}

We thank Mr. Shambhu Bahadur Shrestha, the entomology technician for his help in collecting and identifying sandflies in the field. We also thank Dr. Richard Culleton for critically reading this manuscript. Kishor Pandey is supported by a scholarship from the Japanese Government Ministry of Education, Science, Sports and Culture (MONBUSHO). The present study was supported by the National Bio-Resource Project (NBRP) of MEXT, Japan.

\section{References}

Aransay AM, Scoulica E, Tselentis Y (2000) Detection and identification of Leishmania DNA within naturally infected sand flies by seminested PCR on minicircle kinetoplastic DNA. Appl Environ Microbiol 66: 1933-1938

Basselin M, Badet-Denisot MA, Robert-Gero M (1998) Modification of kinetoplast DNA minicircle composition in pentamidine-resistant Leishmania. Acta Tropica 1998:70:43-61

Bettini S, Gramiccia M, Gradoni L, Atzeni MC (1986) Leishmaniasis is Sardinia: II. Natural infection of Phlebotomus pernicious Newstead, 1911, by Leishmania infantum Nocolle, 1908, in the province of Cagliari. Trans R Soc Trop Med Hyg 80: 458-459

Choi CM, Lerner EA (2001) Leishmaniasis as an emerging infection. J Invest Dermatol Symp Proc 6: 175-182

Cordoba-Lanus E, De Grosso ML, Pinero JE, Valladares B, Salomon OD (2006) Natural infection of Lutzomyia neivai with Leishmania spp. in northwestern Argentina. Acta Trop 98: 1-5

Desjeux P (1996) Leishmaniasis. Public health aspects and control. Clin Dermatol 14: 417-423

Dinesh DS, Kar SK, Kishore K, Palit A, Verma N, Gupta AK, Chauhan DS, Singh D, Sharma VD, Katoch VM (2000) Screening sandflies for natural infection with Leishmania donovani, using a non-radioactive probe based on the total DNA of the parasite. Ann Trop Med Parasitol 94: 447-451

Gradoni L, 2002 The diagnosis of canine leishmaniasis. Canine Leishmaniasis: moving towards a solution, Proocedings of the Second International Canine Leishmaniasis Forum Sevilla, Spain, pp. 7-14

Hassan MM, Elraba FMA, Ward RD, Maingonc RDC, Elnaiem DA 2004 Detection of high rates of in-village transmission of Leishmania donovani in eastern Sudan. Acta Tropica 92: 77-82

Kato H, Uezato H, Katakura K, Calvopiña M, Marco JD, Barroso PA, Gomez EA, Mimori T, Korenaga M, Iwata H, Nonaka S, Hashiguchi Y (2005) Detection and identification of Leishmania species within naturally infected sand flies in the andean areas of ecuador by a polymerase chain reaction. Am J Trop Med Hyg 72: 87-93

Killick-Kendrick R (1999) The biology and control of phlebotomine sand flies. Clin Dermatol 17: 279-289

Kumar V, Bimal, S, Kesari S, Kumar AJ, Bagchi AK, Akbar MA, Kishore K, Bhattacharya SK and Das P (2005) Evaluation of a dot-immunoblot assay for detecting leishmanial antigen in naturally infected Phlebotomusargentipes (Diptera: Psychodidae). Ann Trop Med Parasitol 99( 4): 371-376

Lewis DJ (1987) Phlebotomine sandflies (Diptera: Psychodidae) from the oriental region. Syst Entomol 12: $163-180$

Maroli M, Gramiccia M, Gradoni L, Troiani M, Ascione R (1994) Natural infection of Phlebotomus perniciosus with MON 72 zymodeme of Leishmania infantum in the Campania region of Italy. Acta Trop 57: 333-335

Ministry of Health, Nepal (2005/2006) Annual Report. Kala-azar Control. Kathmandu: His Majesty's Government of Nepal, Ministry of Health, Directorate of Health Services, Epidemiology and Disease Control.

Mukherjee S, Hassan MQ, Ghosh A, Ghosh KN, Bhattacharya A, Adhya S (1997) Short report: Leishmania DNA in Phlebotomus and Sergentomyia species during a kala-azar epidemic. Am J Trop Med Hyg 57: 423-425

Palit A, Bhattacharya SK, Kundu SN (2005) Host preference of Phlebotomus argentipes and Phlebotomus papatasi in different biotopes of West Bengal, India. Int J Environ Health Res 15: 449-454

Pandey K, Yanagi T, Pandey BD, Mallik AK, Sherchand JB, Kanbara H (2007) Characterization of Leishmania isolates from Nepalese patients with visceral leishmaniasis. Parasitol Res 100: 1361-1369

Ready PD, Smith DF, Kellick-Kendrick R (1988) DNA hybridization on squash-blotted sandflies to identify both Phlebotomous papatasi and infecting Leishmania major. Med Vet Entomol 2: 109-11

Salotra P, Sreenivasm G, Pogue GP, Lee N, Nakhasi HL, Ramesh V, Negi NS (2001) Development of a species-specific PCR assay for detection of Leishmania donovani in clinical samples from patients with Kala-azar and Post-Kala-azar dermal leishmaniasis. J Clin Microbiol 39:849-854

Symth AJ, Ghoshm A, Hassanm MQ, Basu D, De Bruijn MHL, Adhya S, Mallik KK, Barker DC (1992) Rapid and sensitive detection of Leishmania 
kinetoplast DNA from spleen and blood samples of kala-azar patients. Parasitology 105:183-192

\section{Figure Legends}

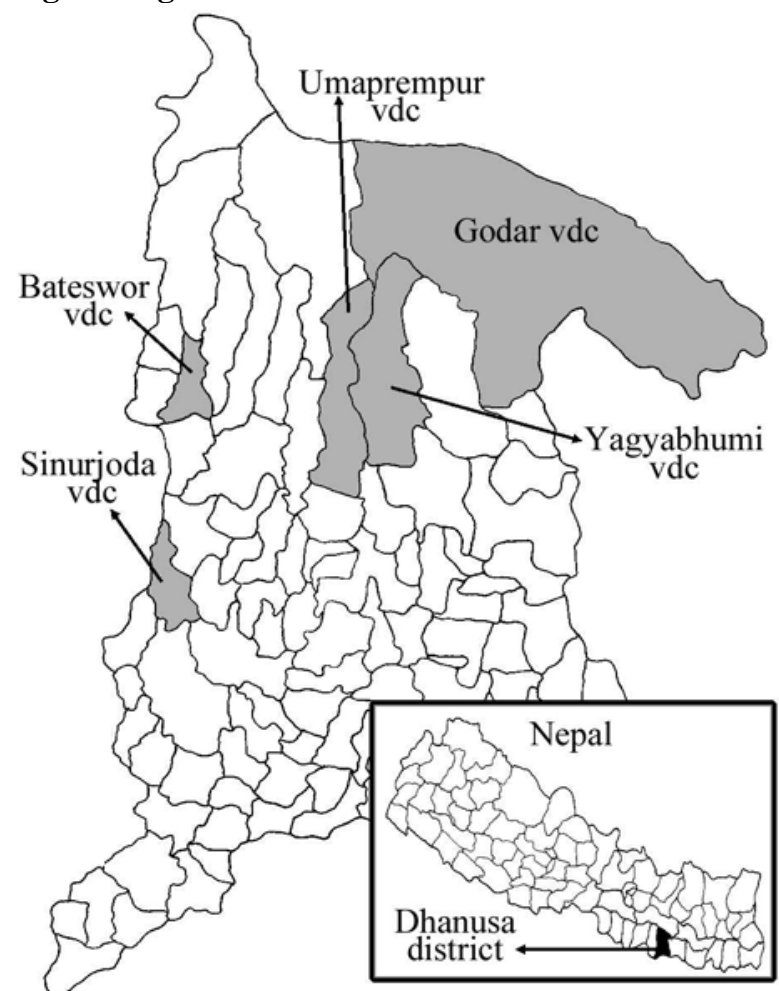

Figure 1. Collection sites of sandflies in Nepal.

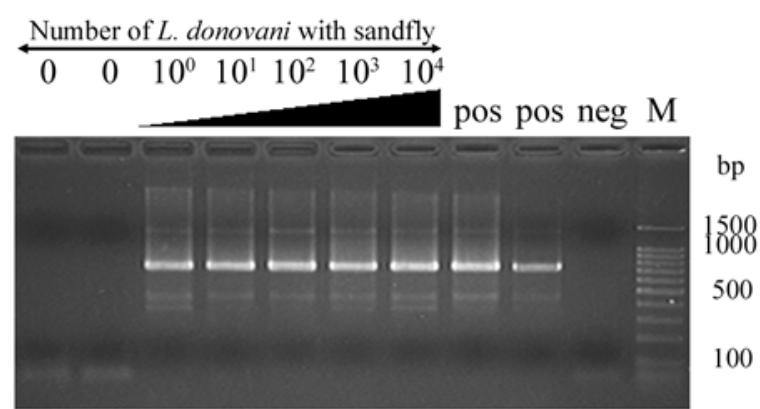

Figure 2. Sensitivity of PCR from DNA extracted from different numbers of Leishmania donovani parasite mixed with the whole bodies of male sandflies. PCR amplification was carried out on DNA was extracted from male sandflies crushed in the presence of $0,1,10,100$, 1000, 10000 L. donovani parasites . M, 100 bp DNA ladder marker; pos, DNA extracted from $L$. donovani cultured parasite as a positive template; neg, distilled water as a negative template solution.

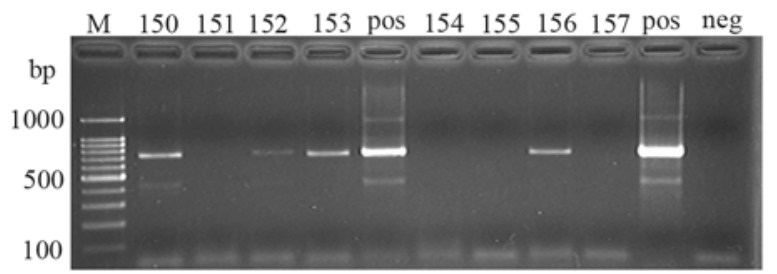

Figure 3. Representative PCR-positive samples from infected and noninfected sandflies with Leishmania spp. M, 100 bp DNA ladder marker; PCR from field-isolated $P$. argentipes (150,152, 13 and 156 are positive; 151, 154, 155 and 157 are negative); pos, DNA extracted from $L$. donovani cultured parasite as a positive template; neg, distilled water as a negative template solution.

Table 1. Female sandflies collected each year

\begin{tabular}{ccccc}
\hline Year & Phlebotomus argentipes & P. papatasi & Sergentomyia spp. & Total \\
\hline 2004 & 22 & 16 & 7 & 45 \\
2005 & 2 & 123 & 23 & 148 \\
2006 & 377 & 63 & 38 & 478 \\
\hline Total & 401 & 202 & 68 & 671 \\
\hline
\end{tabular}

Table 2. Infective rate of sandflies with Leishmania parasites in different villages

\begin{tabular}{lccccccc}
\hline Year & Phlebotomus argentipes & \multicolumn{2}{c}{ P. papatasi } & \multicolumn{3}{c}{ Sergentomyia spp. } \\
\hline \multirow{2}{*}{ Bateshwor } & $9 / 168$ & $(5.4 \%)$ & $0 / 43$ & $(0 \%)$ & $0 / 16$ & $(0 \%)$ \\
Godar & $0 / 2$ & $(0 \%)$ & $0 / 55$ & $(0 \%)$ & $0 / 19$ & $(0 \%)$ \\
Sinurjoda & $3 / 29$ & $(10.3 \%)$ & $0 / 20$ & $(0 \%)$ & $0 / 1$ & $(0 \%)$ \\
Umaprempur & n.a. & & $0 / 68$ & $(0 \%)$ & $0 / 4$ & $(0 \%)$ \\
Yagyabhumi & $15 / 202$ & $(7.4 \%)$ & $0 / 16$ & $(0 \%)$ & $0 / 28$ & $(0 \%)$ \\
\hline Total & $27 / 401$ & $(6.7 \%)$ & $0 / 202$ & $(0 \%)$ & & $0 / 68$ & $(0 \%)$ \\
\hline
\end{tabular}

n.a. indicates that no sandfly was collected. 
Table 3. Comparison of Leishmania spp. infections in sandfly

\begin{tabular}{|c|c|c|c|c|c|}
\hline Infection rate & Leishmania spp. & Sandfly spp. & Country & Reference & method \\
\hline $27 / 402(6.7 \%)$ & Leishmania spp. ${ }^{a}$ & P. argentipes & Nepal & Present study & $\begin{array}{l}\text { PCR assay } \\
\text { from sandfly without dissection }\end{array}$ \\
\hline $14 / 435(3.4 \%)$ & L. donovani & P. argentipes & India & Kumar et al 2005 & dot-immunoblot assay \\
\hline $9 / 572(1.6 \%)$ & L. donovani & P. orientalis & Sudan & Hassan et al 2004 & $\begin{array}{l}\text { PCR assay from dissected } \\
\text { sandfly }\end{array}$ \\
\hline
\end{tabular}

${ }^{\text {a Among }} 27$ samples, 15 were confirmed as L. donovani by nucleotide sequencing. The remaining 12 were uncharacterized. 